\title{
Problem of Cognitive Rehabilitation of Patients with Neurosurgical Pathology of the Brain
}

\author{
Ivanova $\mathrm{NE}^{1 *}$, Efimova $\mathrm{M} \mathrm{Yu}^{1}$, Makarov $\mathrm{AO}^{2}$, Tereshin $\mathrm{AE}^{2}$, Karyagina $\mathrm{MV}^{2}$, Reshetnic $\mathrm{DA}^{2}$ and \\ Ivanov $\mathrm{A} \mathrm{Yu}^{3}$
}

${ }^{1}$ National Medical Research Center named after VA Almazov, Ministry of Health of the Russian Federation, Saint-Petersburg, Russia

${ }^{2}$ Nikolaev Hospital, Saint-Petersburg, Russia

${ }^{3}$ St. Petersburg State Pediatric Medical University; Ministry of Health of the Russian Federation, Saint-Petersburg, Russia

KEYWORDS: Cognitive impairment; Neurosurgical pathology; Rehabilitation

\section{EDITORIAL}

The problem of diagnosis and treatment of cognitive impairment - one of the actual problems of neurorehabilitation after neurological and neurosurgical diseases.Neurosurgical diseases of the brain (brain injury, neuro-oncology, cerebrovascular pathology) are the most important cause of disability in the population of developed countries, leading not only to paresis, hypesthesia, discoordination, but also cognitive disorders. The role of ageing in the formation of cognitive deficiency in focal brain lesions has not been definitively determined yet.

The study was based on the results of cognitive rehabilitation of 165 patients, the average age $48,28 \pm 18,79$ years, operated for focal brain lesions. The structure, severity and dynamics of higher brain function disorders were evaluated. The influence of such factors as the form of neurosurgical pathology, limitation period and traumatism of surgical intervention, educational level and psychoemotional status of patients were considered. An evaluation of the effectiveness of the use of traditional methods of neuropsychological correction in combination with drug and physical therapy, as well as computer programs of cognitive training were compared.

The most common symptoms of cognitive deficits for all types of neurosurgical pathology were identified: fixation hypomnesia, pathological inertia of thinking and decreased attention. The interrelation of the right-hand localization of the lesion with emotional and personal disorders and disorders of spatial thinking, left- hand localization-with violations of verbal and logical thinking is clarified $(\mathrm{p}<0.05)$. It is proved that the development of depression exacerbates existing cognitive deficits $(\mathrm{p}<0.05)$.

At that neuropsychological correction in patients with nontraumatic hemorrhage and brain tumor are more effective than constrictive and occluzimi lesions of the vessels of the carotid system and traumatic brain injury $(\mathrm{p}<0.05)$. The expediency of the most active cognitive rehabilitation, including neuropsychological correction and computer programs Scientific brain training PRO, in the first 6 months after surgical treatment $(\mathrm{p}<0.05)$.

\section{CONCLUSION}

The structure and features of cognitive impairment in neurosurgical patients were studied. The influence on their structure and severity, as well as on the effectiveness of rehabilitation measures of a number of factors, including the localization and nature of the pathological process, the volume and timing of surgery, the level of education and psycho-emotional status. The results can be used to determine the rehabilitation prognosis.
Quick Response Code:

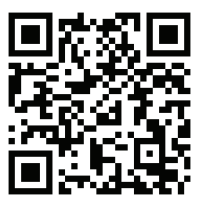

Address for correspondence: Ivanova NE, National Medical Research Center named after VA Almazov, Ministry of Health of the Russian Federation, Saint-Petersburg, Russia

Received: July 29, 2019 Published: August 12, 2019

How to cite this article: Ivanova NE. Problem of Cognitive Rehabilitation of Patients with Neurosurgical Pathology of the Brain. 2019 - 1(1) OAJBS.ID.000101. DOI: $10.38125 / O A J B S .000101$ 
The comparative effectiveness of the use of medical, physiotherapeutic and computer techniques in the cognitive rehabilitation of neurosurgical patients was analyzed. Based on the results of a comprehensive analysis of the effectiveness of rehabilitation measures, an algorithm of cognitive rehabilitation of neurosurgical patients was developed.

The structure and severity of cognitive impairment in neurosurgical pathology depend on the etiology of the pathological process, localization and size of the pathological focus, psychoemotional status and educational level of patients. Age does not directly affect the severity of cognitive deficits.
The basis of cognitive rehabilitation of neurosurgical patients is neuropsychological correction. Its effectiveness is maximum in the first 6 months after surgical treatment and further decreases over time. Application in complex cognitive rehabilitation of neurosurgical patients drugs Akatinol Memantine and Ceraxon, physiotherapy (photochromotherapy and transcranial electromagnetic stimulation) and computer technology effectively and safely when taking into account indications and contraindications, compliance with the optimal timing and techniques of performance of procedures. 